\title{
Enhancement of carotenoids biosynthesis in Chlamydomonas reinhardtii by nuclear transformation using a phytoene synthase gene isolated from Chlorella zofingiensis
}

\author{
Baldo F. Cordero • Inmaculada Couso • Rosa León • \\ Herminia Rodríguez $\cdot$ M. Ángeles Vargas
}

Received: 11 November 2010/Revised: 15 March 2011 /Accepted: 5 April 2011 /Published online: 26 April 2011

(C) The Author(s) 2011. This article is published with open access at Springerlink.com

\begin{abstract}
The isolation and characterization of the phytoene synthase gene from the green microalga Chlorella zofingiensis (CzPSY), involved in the first step of the carotenoids biosynthetic pathway, have been performed. $C z P S Y$ gene encodes a polypeptide of 420 amino acids. A single copy of $C z P S Y$ has been found in $C$. zofingiensis by Southern blot analysis. Heterologous genetic complementation in Escherichia coli showed the ability of the predicted protein to catalyze the condensation of two molecules of geranylgeranyl pyrophosphate (GGPP) to form phytoene. Phylogenetic analysis has shown that the deduced protein forms a cluster with the rest of the phytoene synthases (PSY) of the chlorophycean microalgae studied, being very closely related to PSY of plants. This new isolated gene has been adequately inserted in a vector and expressed in Chlamydomonas reinhardtii. The overexpression of $C Z P S Y$ in $C$. reinhardtii, by nuclear transformation, has led to an increase in the corresponding $C z P S Y$ transcript level as well as in the content of the carotenoids violaxanthin and lutein which were 2.0- and 2.2-fold higher than in untransformed cells. This is an example of
\end{abstract}

B. F. Cordero · I. Couso $\cdot$ H. Rodríguez $\cdot$ M. Á. Vargas $(\bowtie)$ Instituto de Bioquímica Vegetal y Fotosíntesis,

Centro de Investigaciones Científicas Isla de la Cartuja,

University of Sevilla and Consejo Superior de Investigaciones

Científicas, Avda. Américo Vespucio no 49,

41092 Sevilla, Spain

e-mail: avargas@us.es

R. León

Departamento de Química y Ciencia de Materiales,

Facultad de Ciencias Experimentales, University of Huelva,

Avda. Fuerzas Armadas s/n,

21071 Huelva, Spain manipulation of the carotenogenic pathway in eukaryotic microalgae, which can open up the possibility of enhancing the productivity of commercial carotenoids by molecular engineering.

Keywords Carotenoids · Chlorella zofingiensis · Phytoene synthase $\cdot$ Transgenic microalgae $\cdot$ Chlamydomonas reinhardtii

\section{Introduction}

Carotenoids are isoprenoids synthesised by all photosynthetic organisms and by some fungi and non-photosynthetic bacteria. In photosynthetic organisms, carotenoids bind to integral membrane proteins of the thylakoid where they participate in light harvesting and in the protection of the photosynthetic apparatus against the photo-oxidative damage (Frank and Cogdell 1996; Varkonyi et al. 2002). Carotenoids are high-value compounds, being used as nutraceuticals and as natural dyes and additives in food, feed, aquaculture, and cosmetic industries. They are considered effective agents for the prevention of a variety of age-related, degenerative and chronic diseases such as cataracts, macular degeneration, atherosclerosis, cardiovascular diseases, and some types of cancer (Dweyer et al. 2001; Demming-Adams and Adams 2002; Guerin et al. 2003; Olmedilla et al. 2003; Stahl and Sies 2005).

In chloroplasts of plants and algae, the carotenoids precursor, geranylgeranyl pyrophosphate (GGPP), is formed by the action of GGPP synthase from isopentenyl pyrophosphate and dimethylallyl pyrophosphate, which are derived from deoxyxylulose 5-phosphate pathway. GGPP is not only the precursor for carotenoids but also participate in 
the synthesis of other terpenoid compounds, such as phytol, plastoquinones, and tocopherols. The condensation of two GGPP molecules produces the first carotene, phytoene, catalyzed by PSY (Fig. 1). Phytoene is desaturated by phytoene and $\zeta$-carotene desaturases (PDS and ZDS) and isomerized by 15 -cis- $\zeta$-carotene isomerase (Z-ISO) (Chen et al. 2010) and carotene isomerase (CRTISO) to form the linear all trans-lycopene. The cyclation of lycopene by lycopene $\varepsilon$-cyclase (LCYe) and lycopene $\beta$-cyclase (LCYb) introduces $\varepsilon$ - and $\beta$-ionone end groups, respectively, yielding $\alpha$ - and $\beta$-carotenes. $\alpha$ - and $\beta$-carotene are hydroxylated into lutein and zeaxanthin, respectively. Two $\mathrm{P} 450$ hydroxylases (P450b-CHY and $\mathrm{P} 450 \mathrm{e}-\mathrm{CHY}$ ) are active in $\alpha$-carotene hydroxylation. $\mathrm{P} 450 \mathrm{~b}-\mathrm{CHY}$ is also active in $\beta$-carotene hydroxylation, together with two nonheme di-iron hydroxylases (CHYb). Zeaxanthin epoxidase (ZEP) and violaxanthin de-epoxidase (VDE) catalyze the interconversion of zeaxanthin and violaxanthin (Kim et al. 2009; Sandmann et al. 2006). A limited number of organisms including some green algae as Haematococcus pluvialis and Chlorella zofingiensis can synthesize astaxanthin from $\beta$-carotene by the action of a ketolase/ oxygenase (BKT) and the hydroxylase ( $\mathrm{CHYb}$ ) (Fan et al. 1995; Huang et al. 2006; Li et al. 2008a).

Although the regulatory mechanisms that control carotenoid biosynthesis are poorly understood, there is

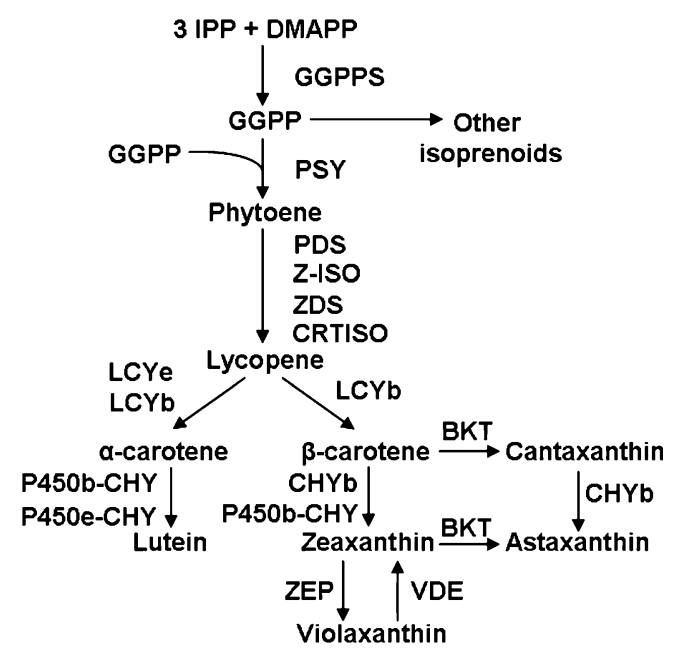

Fig. 1 Schematic diagram of the carotenoid biosynthetic pathway in plants and microalgae. Phytoene synthase (PSY) catalyses the first step in the carotenoid specific pathway, which leads the carbon flux towards carotenes and xantophylls production. IPP isopentenyl pyrophosphate, DMAPP dimethylallyl pyrophosphate, GGPP geranylgeranyl pyrophosphate, GGPPS geranylgeranyl pyrophosphate synthase, $P D S$ phytoene desaturase, Z-ISO 15 -cis- $\zeta$-carotene isomerase, $Z D S \zeta$-carotene desaturase, CRTISO carotene isomerase, $L C Y b$ lycopene $\beta$-cyclase, LCYe lycopene $\varepsilon$-cyclase, $\mathrm{P} 450 \mathrm{~b}-\mathrm{CHY}$ cytochrome P450 $\beta$-hydroxylase, P450e-CHY cytochrome P450 $\varepsilon$ hydroxylase, $C H Y b$ carotene $\beta$-hydroxylase, $B K T \beta$-carotene oxygenase, $Z E P$ zeaxanthin epoxidase, $V D E$ violaxanthin de-epoxidase abundant evidence to indicate that the reaction catalyzed by PSY, first committed step of the carotenoid synthesis, is an important control point for the regulation of carbon flux into and through the pathway (Fraser et al. 2002; Sandmann et al. 2006). The high economic value of carotenoids as nutritional sources of vitamin A and healthpromoting compounds has stimulated research to increase carotenoid biosynthesis in crop plants through genetic manipulation of the pathway. Overexpression of bacterial or plant phytoene synthase genes in higher plants has resulted in a significant increase in total carotenoid levels in tomato and Hongkong kumquat (Fortunella hindsii) Swingle fruit (Fray et al. 1995; Fraser et al. 2002; Zhang et al. 2009), Canola and Arabidopsis seeds (Shewmaker et al. 1999; Lindgren et al. 2003), rice endosperm (Paine et al. 2005), potato tuber (Ducreux et al. 2005), and carrot (Baranski 2008). In microalgae, only a few works describe genetic manipulation of the carotenogenic pathway. Silencing, via RNA interference, of PDS in Dunaliella (Sun et al. 2007) and Chlamydomonas (Vila et al. 2007), silencing of the Chlamydomonas PSY gene by artificial microRNAs (Molnar et al. 2009), transformation of H. pluvialis with a modified PDS (Steinbrenner and Sandmann 2006), and the production of a new ketocarotenoid in Chlamydomonas through the expression of a foreign $\beta$-carotene oxygenase (BKT) gene (León et al. 2007) are some examples. The chlorophyta $C$. zofingiensis accumulates high amounts of astaxanthin and lutein (Del Campo et al. 2004; Sun et al. 2008) and is considered as a model organism to study the regulation of the carotenoids biosynthetic pathway, since it produces the primary carotenoid lutein as well as the secondary carotenoid astaxanthin. However, only the carotenogenic genes BKT (Huang et al. 2006), PDS (Huang et al. 2008), $C H Y b$ (Li et al. 2008b), and $L C Y b$ (Cordero et al. 2010) have been isolated and characterized in this microalga until now. In addition, nuclear transformation in this microalga has never been accomplished. Chlamydomonas reinhardtii is the first and best studied transformed chlorophyte, it grows at high rates, and its nuclear genetic manipulation is easy and well established. This makes $C$. reinhardtii a good candidate to express foreign carotenogenic genes for the biotechnological production of commercially interesting carotenoids and for carrying out basic metabolic and regulatory studies of the pathway (León et al. 2004).

In the present work, we report the isolation and characterization of the $P S Y$ gene from $C$. zofingiensis, as well as its ability to convert two GGPP molecules into phytoene. Moreover, this novel gene has been inserted in an adequate vector and expressed in $C$. reinhardtii. This is an example of the overexpression of an exogenous gene (PSY) in an eukaryotic microalgae, which can be an interesting tool for the massive production of carotenoids in transgenic microalgae by genetic engineering. 


\section{Materials and methods}

Strains and culture conditions

The green microalga strain C. zofingiensis SAG 211-14 was obtained from the Culture Collection of Göttingen University (SAG, Germany). This microalga was grown photoautotrophically in Arnon medium (Arnon et al. 1974) modified to contain $4 \mathrm{mM} \mathrm{K}_{2} \mathrm{HPO}_{4}$ and $20 \mathrm{mM} \mathrm{NaNO}_{3}$, at $25^{\circ} \mathrm{C}$ under continuous illumination $(50 \mu \mathrm{mol}$ photons $\mathrm{m}^{-2} \mathrm{~s}^{-1}$ ). The light intensity was measured at the surface of the flasks using a LI-COR quantum sensor (model L11905B, Li-Cor, Inc. Lincoln, NE, USA). The liquid cultures were continuously bubbled with air supplemented with $1 \%$ $(v / v) \mathrm{CO}_{2}$ as the only source of carbon. $C$. reinhardtii cellwall-deficient strain 704 was kindly provided by Dr. Roland Loppes (Loppes et al. 1999) and cultured mixotrophically in either liquid or agar solidified Tris-acetate phosphate (TAP) medium (Gorman and Levine 1965) at $25^{\circ} \mathrm{C}$ under a continuous irradiance of $50 \mu \mathrm{mol}$ photons $\mathrm{m}^{-2} \mathrm{~s}^{-1}$. Escherichia coli DH5 $\alpha$ and BL21 (DE3) strains were used as the hosts for DNA manipulation and for heterologous expression of PSY gene, respectively.

For the analysis of transformants, cells were grown in Erlenmeyer flasks of $100 \mathrm{~mL}$ capacity at $25^{\circ} \mathrm{C}$ under continuous illumination $\left(50 \mu \mathrm{mol}\right.$ photons $\left.\mathrm{m}^{-2} \mathrm{~s}^{-1}\right)$ in liquid TAP medium.

Genomic DNA and RNA isolation and cDNA preparation

DNA and total RNA were isolated using DNeasy Plant Mini Kit and RNeasy Plant Mini Kit (Qiagen, Düsseldorf, Germany), respectively. For genomic DNA isolation for PCR screening of transformants from $C$. reinhardtii, a loopful of cells was scrapped from a plate and resuspended in $150 \mu \mathrm{L}$ of cold distilled water and $350 \mu \mathrm{L}$ of a buffered solution containing $50 \mathrm{mM}$ Tris- $\mathrm{HCl}, \mathrm{pH} 8,0.3 \mathrm{M} \mathrm{NaCl}$, $5 \mathrm{mM}$ EDTA, and 2\% SDS. The DNA isolation was performed by phenol-chloroform-isoamyl alcohol (50:48:2) extraction and selective precipitation with ethanol, according to previously described protocols (Anwaruzzaman et al. 2004). For quantitative real-time PCR analysis (qRT-PCR), first-strand cDNA synthesis was obtained from total RNA treated with DNase as recommended by the manufacturer, by using the SuperScript First-Strand Synthesis System (Invitrogen, Barcelona, Spain) primed with oligo $(\mathrm{dT})_{18}$ according to the manufacturer's instructions.

Cloning of C. zofingiensis PSY cDNA and genomic gene

For isolating the cDNA clone coding for the C. zofingiensis PSY homologue, amino acid sequences deduced from previously cloned PSY genes from different kinds of algae, cyanobacteria, and plants were aligned. Highly conserved regions were identified, and different pairs of degenerated primers were designed. The PCR product was cloned in the pGEM-T vector (Promega, Madison, WI, USA) according to the manufacturer's manual and then sequenced. The cDNA fragment obtained corresponding to partial PSY clone provided sequence information for the designing of genespecific primers for amplification of $5^{\prime}$ and $3^{\prime} \mathrm{cDNA}$ ends by RACE-PCR. All reactions were performed with kits according to the manufacturer's instructions (Smart RACE cDNA Amplification Kit, Clontech, Mountain View, CA, USA). 5' and 3' RACE products were cloned into pGEM-T vector and sequenced. Specific primers were synthesized for genomic DNA amplification based on cDNA sequence. The primers sets used in this study are listed in Table 1.

Nucleotide sequence accession numbers

The $C z P S Y$ cDNA and genomic DNA sequences have been registered in the EMBL database (EMBL, FR670783 and EMBL, FR670784, respectively).

\section{Sequencing and phylogenetic analysis}

The deduced amino acid sequence of the $C$. zofingiensis PSY was compared with other PSY sequences of algae, plants, cyanobacteria, and bacteria. The sequence analysis and alignments were done with CLUSTAL_W software. The deduced amino acid sequence was subjected to the ProtParam application at the ExPASy server (Gasteiger et al. 2005) for physical and chemical parameters, program Predotar v. 1.03 (Caboche 2003) for the prediction of possible plastid localization, and ChloroP 1.1 server (Emanuelsson et al. 1999) for the identification of a chloroplast transit peptide. For transmembrane analysis, ProtScale (Gasteiger et al. 2005) and TopPred (von Heijne 1992) servers were used. The construction of a phylogenetic tree was performed in MEGA4 (Tamura et al. 2007) using the UPGMA method.

Southern blot analysis

Genomic DNA was digested with HincII and HindIII, which showed one and no recognition site, respectively, in the probed region of the $P S Y$ gene. The probe was prepared by amplifying genomic DNA with the primers Czpsy-S-F and Czpsy-S-R, resulting in a 752-bp fragment of $C z P S Y$ gene. The digested DNA was transferred to a Hybond-N membrane (GE Healthcare, Little Chanfont, UK) by capillary transfer and hybridized with the ${ }^{32} \mathrm{P}$ labelled DNA probe at both low and high stringency overnight. After hybridization, the radioactivity of the membrane was monitored by the Cyclone Phosphor System (Perkin-Elmer, Waltham, MA, USA). 
Table 1 Nucleotide sequences of primer pairs used for PCR amplification

\begin{tabular}{|c|c|}
\hline Primer & Sequence $\left(5^{\prime} \rightarrow 3^{\prime}\right)$ \\
\hline \multicolumn{2}{|c|}{ Partial PSY fragment } \\
\hline psy-1F & GAYATGATHGARGGNATG \\
\hline psy-1R & AARTTRTCRTARTCRTT \\
\hline \multicolumn{2}{|l|}{ 5'and 3' RACE } \\
\hline GSP-F & ATGAATTTAGTCAAGTCACGG \\
\hline GSP-R & TTTGGGCACATAAGCACG \\
\hline NGSP-F & CTGGATGAGAAGGCAAGG \\
\hline NGSP-R & CCTTGCCTTCTCATCCAG \\
\hline \multicolumn{2}{|c|}{ Genomic DNA amplification } \\
\hline psy-2F & ACATGGGGGCGAATTTGTTGGT \\
\hline psy-2R & CCCGTGCCTGCTTAGGAGTCAT \\
\hline psy-3F & ATGCGGTGCGGTTACCAGTGAA \\
\hline psy-3R & ACCTGTCATCTATCTTGCCTGT \\
\hline psy-4F & ACAGGCAAGATAGATGACAGGT \\
\hline psy-4R & CTCTCCAAAGTTAAGATGAACA \\
\hline psy-5F & TGTTCATCTTAACTTTGGAGAGTTT \\
\hline psy-5R & AGCACGCATTACAAGGCTCC \\
\hline \multicolumn{2}{|c|}{ PCR for probe preparation } \\
\hline Czpsy-S-F & ATGGATTTAGTCAAGTCACGG \\
\hline Czpsy-S-R & TTTGGGCACATAAGCACG \\
\hline \multicolumn{2}{|c|}{ Genetic complementation-pQE- $80 \mathrm{~L}^{\mathrm{a}}$} \\
\hline pQE-psy-F & ggatccATGGCGTCGTTTAGCACCAGG \\
\hline pQE-psy-R & ggtaccTTAGCTGCTGGTTGCCGCAGC \\
\hline \multicolumn{2}{|c|}{ PCR for Chamydomonas reinhardtii transformation ${ }^{\mathrm{b}}$} \\
\hline CzpsyCr105-F & cgcctcgagTATGGCGTCGTTTAGCACCAG \\
\hline CzpsyCr105-R & cgcgaattcTTAGCTGCTGGTTGCC \\
\hline \multicolumn{2}{|l|}{ CzPSY expression } \\
\hline RT-Czpsy-F & CACCAGGTTGTCAGAGTCCA \\
\hline RT-Czpsy-R & ACTAGTGTGTTGCTGACTCT \\
\hline \multicolumn{2}{|l|}{ CrPSY expression } \\
\hline RT-Crpsy-F & CACTCGCGCCCGGCAATACTT \\
\hline RT-Crpsy-R & CCACGGGCAGCGACACCATC \\
\hline \multicolumn{2}{|l|}{ CBLP expression } \\
\hline RT-cblp-F & CGCCACCCAGTCCTCCATCAAGA \\
\hline RT-cblp-R & CTAGGCGCGGCTGGGCATTTAC \\
\hline
\end{tabular}

$F$ forward, $R$ reverse

${ }^{a}$ Bam HI and KpnI sites (lowercases underlined) were added for cloning the gene into the corresponding cut sites of $\mathrm{pQE}-80 \mathrm{~L}$ vector

${ }^{\mathrm{b}} \mathrm{XhoI}$ and EcoRI sites (lowercases underlined) were added for cloning the gene into the corresponding cut sites of pSI105 plasmid

Functional analysis of $C z P S Y$ cDNA by heterologous expression in E. coli

The CzPSY ORF was amplified by PCR with the primers pQE-psy-F and pQE-psy-R, which were designed to contain BamHI and KpnI restriction sites, respectively, and cloned between the $B a m \mathrm{HI}$ and $K p n I$ restriction sites of the pQE-80L expression vector (Qiagen), resulting in plasmid pQE-CzPSY, which carries ampicillin resistance. Plasmid pAC-85b, a gift from Prof. Cunningham, contained the carotenoid pathway genes responsible for the synthesis of $\beta$-carotene except PSY gene (crtE, crtI, and $\operatorname{crt} Y$ of Erwinia herbicola) (Cunningham and Gantt 2007). Transformation of $E$. coli BL21 (DE3) with either pAC-85b (carrying chloramphenicol resistance) or pAC-85b and one of the two plasmids, pQE-CZPSY or pQE-80L (empty vector), was made by electroporation. Transformed cells were plated on Luria-Bertani solid medium (Sambrook et al. 1989), supplemented with $100 \mu \mathrm{g} \mathrm{mL}^{-1}$ ampicillin and/ or $40 \mu \mathrm{g} \mathrm{mL} \mathrm{m}^{-1}$ chloramphenicol, and grown at $37^{\circ} \mathrm{C}$ for 1 day. The inducer isopropyl- $\beta$-D-1-thiogalactopyranoside was added at a final concentration of $1 \mathrm{mM}$.

\section{Chlamydomonas nuclear transformation}

The complete coding region of $C Z P S Y$ was amplified by PCR with primers CzpsyCr105-F and CzpsyCr105-R, which were designed to contain $\mathrm{XhoI}$ and EcoRI restriction sites respectively, and cloned into pGEM-T vector (Promega), which carries ampicillin resistance. The PCR product was digested with $X h o \mathrm{I}$ and EcoRI and cloned in the pSI105-Tp1 vector, resulting in the plasmid pSI105-Tp1psy that was used to transform Chlamydomonas cells. The plasmid pSI105-Tp1 is based on the plasmid pSI104-PLK (León et al. 2007), derived from the pSI103 (Sizova et al. 2001), in which the aphVIII gene from Streptomyces rimosus, coding for an aminoglycoside 3'phosphotransferase that confers resistance to the antibiotic paromomycin, is expressed under the control of the strong constitutive promoters $r b c S 2$ and $h s p 70 A$ and terminated by the $3^{\prime}$ untranslated region of $r b c S 2$. The construction pSI105-Tp1 also carries a second expression cassette driven by the same two constitutive promoters and terminator region. This second cassette carries the transit peptide of RuBisCO small subunit $(r b c S 2)$ to target the final peptide into the chloroplast stroma and also carries a polilynker region in which the cDNA from C. zofingiensis PSY was subcloned in frame with the transit peptide sequence.

Nuclear transformation was carried out using the glass beads method of Kindle (1990) with some modifications (León et al. 2007). C. reinhardtii cells grown to about $10^{7}$ cells $\mathrm{mL}^{-1}$ were harvested by centrifugation and resuspended in fresh TAP medium to obtain a 100-fold concentrated cell suspension. The concentrated cell suspension $(0.6 \mathrm{~mL})$ was added to a conical tube containing $0.3 \mathrm{~g}$ of sterile glass beads (Ø $0.4-0.6 \mathrm{~mm}$ ), $0.2 \mathrm{~mL}$ of $20 \%$ polyethylene glycol (MW8000) and $1 \mu \mathrm{g}$ of the desired plasmid. Cells were vortexed and resuspended in $50 \mathrm{~mL}$ of fresh sterile TAP medium where they were incubated in the dark overnight. After this incubation, cells were centrifuged 
and spread onto solid TAP medium with paromomycin $\left(30 \mu \mathrm{g} \mathrm{mL}^{-1}\right)$. Transformed colonies were visible after 4 to 5 days.

\section{Quantitative RT-PCR}

The mRNA relative abundance of endogenous $C$. reinhardtii PSY and foreign C. zofingiensis PSY was examined by qRT-PCR on an IQ5 Real-Time PCR Detection System (BioRad, Hercules, CA, USA), according to Cordero et al. (2010). In each experiment, a series of standard dilutions containing a specific concentration of a PCR fragment or a cDNA template was amplified in $20 \mu \mathrm{L}$ of reaction containing $1 \times$ SYBR Green PCR Master Mix (Quantimix Easy SYG kit, BioTools B\&M Labs, Madrid, Spain) and corresponding primers for either PSY from C. zofingiensis or $C$. reinhardtii (Table 1 ). After heating at $95^{\circ} \mathrm{C}$ for $10 \mathrm{~min}$, cycling parameters were: 40 cycles of $95^{\circ} \mathrm{C}$ for $30 \mathrm{~s}, 60^{\circ} \mathrm{C}$ for $30 \mathrm{~s}$, and $72^{\circ} \mathrm{C}$ for $30 \mathrm{~s}$. Finally, the specificity of the qRT-PCR products was confirmed by performing a melting temperature analysis at temperatures ranging from $55^{\circ} \mathrm{C}$ to $95^{\circ} \mathrm{C}$ at $0.5^{\circ} \mathrm{C} / \mathrm{min}$ and also by electrophoresis on a $2 \%$ agarose gel. Data were captured as amplification plots. Transcription levels of the target genes were calculated from the threshold cycle by interpolation from the standard curve. To standardize the results, the relative abundance of $C B L P$ gene, which encodes a Gprotein $\beta$-subunit-like polypeptide (von Kampen et al. 1994), was also determined and used as the internal standard. The complete experiments (RNA isolation, cDNA synthesis followed with qRT-PCR) were repeated twice independently, and the data were averaged.

Analytical methods

\section{Cell concentration and dry weight determinations}

Cell number was determined with a Neubauer hemocytometer. For dry weight measurements, aliquots $(5 \mathrm{~mL})$ of the cell culture were filtered through Whatman GF/C paper (Whatman plc, Kent, UK), washed three times, and dried at $80^{\circ} \mathrm{C}$ for $24 \mathrm{~h}$.

\section{Carotenoid extraction and HPLC analysis}

Total pigments were extracted with $80 \%$ of acetone $(v / v)$ according to León et al. (2005). Then the samples were centrifuged and analysed by HPLC using a Waters Spherisorb ODS2 column $(4.6 \times 250 \mathrm{~mm}, 5 \mu \mathrm{m}$ particle size) (Waters, Mildford, MA, USA). The chromatographic method described by Cordero et al. (2010) was used. Pigments were eluted at a flow rate of $1.0 \mathrm{~mL} \mathrm{~min}^{-1}$ and were detected at $440 \mathrm{~nm}$ using a Waters 2996 photodiode- array detector. Identification of carotenoids was achieved by comparison of the individual characteristic absorption spectrum and the retention time with known standards. Quantification was performed using a calibration curve generated with commercially available carotenoids standards from Sigma-Aldrich (St. Louis, MO, USA) and DHI (Holsholm, Germany).

\section{Results}

Isolation and characterization of the PSY gene and deduced protein from C. zofingiensis

Different pairs of degenerate primers were designed on the basis of the conserved motifs present in PSY from microalgae, cyanobacteria, and plants. A partial cDNA fragment of $1,337 \mathrm{bp}$ was isolated by PCR amplification using degenerate primers (psy-1F and psy-1R) (Table 1). A complete BLAST homologous search in the Genbank database showed that this fragment had enough similarity with the PSY gene from other species, and provided sequence information for designing specific primers for rapid amplification of 5'and 3' cDNA ends (RACE-PCR). This analysis generated a full-length cDNA of 2,944 bp, which contained an ORF of 1,263 bp, 310-nucleotides of 5'untranslated region (UTR), and a long $3^{\prime}$ UTR of 1,340 nucleotides. A typical algal polyadenylation signal TGTAAA (Gruber et al. 1992) was present in the $3^{\prime}$ UTR at 18 nucleotides upstream from the beginning of the poly(A) tail. The predicted protein has 420 amino acids residues, with an estimated molecular weight of $47.64 \mathrm{kDa}$, a theoretical isoelectric point of 8.53 and an instability index of 48.41 (data obtained with ProtParam program).

The differences between the C. zofingiensis PSY gene and the cDNA sequence were compared and revealed the presence of four exons and three introns (Fig. 2).

To determine the copy number of PSY gene in the genome of $C$. zofingiensis, genomic DNA was digested with two different restriction enzymes (either HincII or HindIII) and subjected to Southern blot analysis at different conditions of stringency. Using a 752-bp fragment of CzPSY as a probe, strong hybridization signals have been obtained with both digestions. The digestion with HincII enzyme, which cuts once inside the probe sequence, showed two bands, while digestion with HindIII, with no restriction site in the probe, exhibited only one band (Fig. 3). These results have suggested the presence of a single copy of the PSY gene in the genome of C. zofingiensis.

The BlastP search results demonstrated that the cloned CzPSY showed the highest overall homology sequence with other PSY from green algae, such as Dunaliella salina and Dunaliella sp. (identity, 74\% and similarity, 84\%) and 


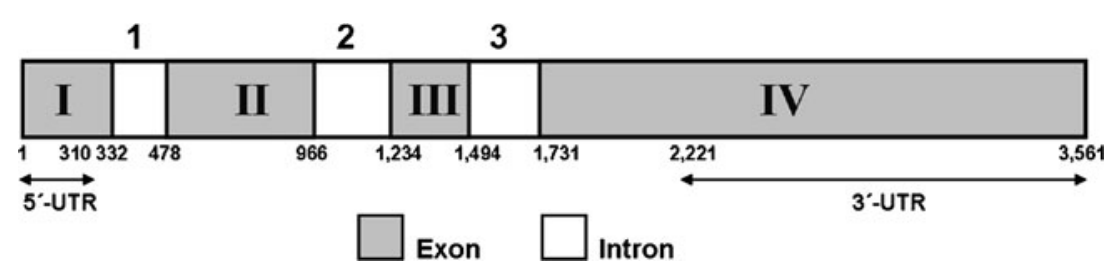

Fig. $2 C z P S Y$ gene organization. The diagram shows exons $(I-I V)$ and introns $(1-3)$ location. The $5^{\prime}$ UTR and 3' UTR sequences are indicated with arrows. Numbers indicate cDNA sequence coordinates (bp)

C. reinhardtii (identity, $70 \%$ and similarity, $84 \%$ ). The GC content of the $C z P S Y$ coding region was $53 \%$, which was lower than that of $D$. salina $(64 \%)$ or of $C$. reinhardtii (59\%). The phylogenetic analysis of the PSY from microalgae, cyanobacteria, plants, and bacteria is illustrated in Fig. 4. Analysis was conducted in MEGA4 (Tamura et al. 2007) using the UPGMA method. The predicted CzPSY forms a cluster with the rest of the microalgae studied, which are phylogenetically closely related to PSY of plants (between $65 \%$ and $70 \%$ of identity and around $75-80 \%$ similarity). The degree of homology was lower with cyanobacterial phytoene synthases (55-60\% identity and 65-70\% similarity). As other algal PSY, CzPSY, was distantly related to bacterial PSY (CRTB), sharing with them only a few conserved motifs and less than $40 \%$ identity.

Since in microalgae and plants PSY is located in the chloroplast, we analysed the CzPSY sequence with two different programs to determine the presence of a signal peptide. The Predotar v. 1.03 program predicted putative plastid localization for the CzPSY, and ChloroP 1.1 server identified an N-terminal chloroplast transit peptide at position 45. Analysis with ProtScale and TopPred servers identified a deduced transmembrane domain of CzPSY located between amino acids 236 and 256 .

Functional analysis of the $C z P S Y$ in $E$. coli

The use of $E$. coli engineered to produce different carotenogenic substrates is an efficient and frequently used method for the functional characterization of the enzymes of the carotenoid biosynthetic pathway (Cunningham and Gantt 2005; Cunningham et al. 1996), due to the complexity of determining the activity of these membrane-associate and low-abundant enzymes.

In order to check the functionality of the recently isolated gene, the full-length ORF of $C z P S Y$ was amplified and cloned into $\mathrm{pQE}-80 \mathrm{~L}$ vector under the control of the $\beta$ galactosidase promoter. The resulting plasmid (pQE$C z P S Y$ ) was introduced in E. coli that carried the plasmid pAC-85b, which contained the carotenogenic genes responsible for the synthesis of $\beta$-carotene as final product except $P S Y$ gene. HPLC analysis of carotenoids extracted from $E$. coli showed that cells containing pAC-85b and pQE-CzPSY produced $\beta$-carotene (Fig. 5). As negative controls, E. coli co-transformed with $\mathrm{pAC}-85 \mathrm{~b}$ or $\mathrm{pAC}-85 \mathrm{~b}$ and empty $\mathrm{pQE}-$ $80 \mathrm{~L}$ were used, resulting in no accumulation of carotenoids. These results confirmed the functionality of $C z P S Y$ gene product, which could catalyze the conversion of two molecules of GGPP into phytoene, as most PSY previously isolated.

Nuclear transformation of $C$. reinhardtii with $P S Y$ gene from $C$. zofingiensis and screening of transformants obtained

The complete coding region of $C Z P S Y$ gene was amplified by PCR and cloned between the XhoI and EcoRI restriction sites of the Chlamydomonas expression vector pSI105-Tp1, resulting in the plasmid pSI105-psy. C. reinhardtii cells a

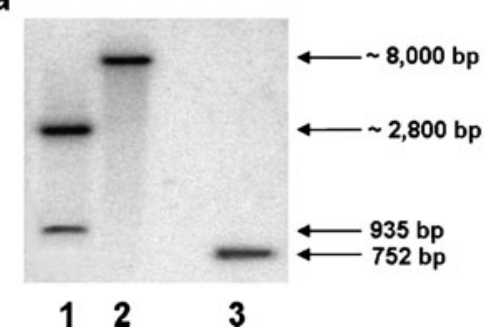

Fig. 3 Southern blot analysis of genomic DNA from C. zofingiensis. a DNA was digested with HincII (lane 1) and HindIII (lane 2), electrophoretically separated on a $0.8 \%$ agarose gel, blotted and hybridized at high stringency with a probe of $752 \mathrm{bp}$ of the PSY gene

b

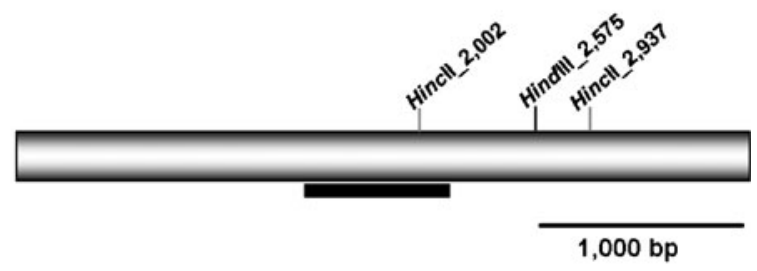

amplified by PCR. A plasmid containing the $P S Y$ gene was used as a positive control (lane 3). b HincII and HindIII restriction sites present in the $C z P S Y$ gene. The black bar indicates the probe location 


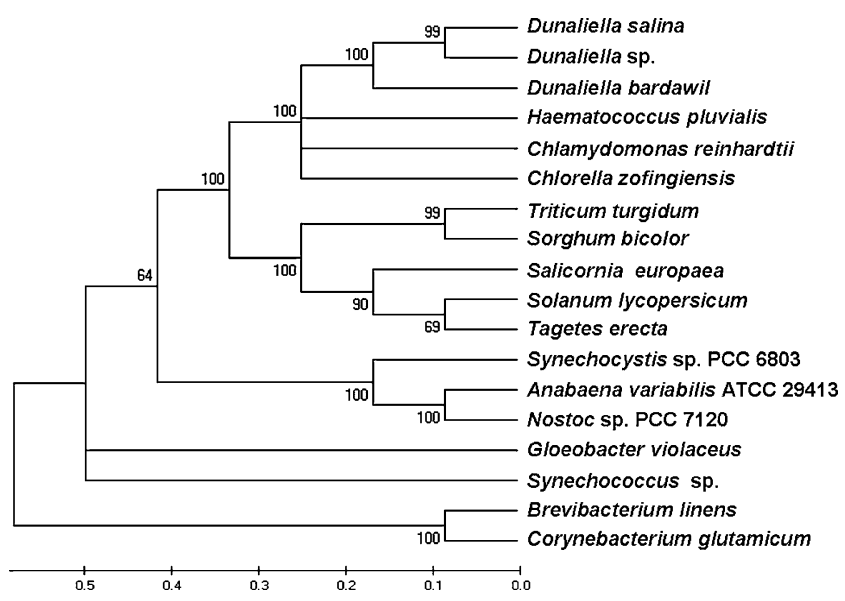

Fig. 4 UPGMA tree analysis of the indicated plant, algal, cyanobacterial, and bacterial PSY amino acid sequences. Analysis was performed in MEGA4 (Tamura et al. 2007). The GenBank accession numbers for other species are as follows: Dunaliella salina, AAT46069; Dunaliella sp., ABE97388.1; Dunaliella bardawil, AAB51287.1; Haematococcus pluvialis, AAW28851.1; Chamydomonas reinhardtii, XP 001701192.1; Triticum turgidum, ACQ59152.1; Sorghum bicolor, ACY70869; Salicornia europaea, AAX19898.1; Solanum lycopersicum, ABM45873.1; Tagetes erecta, AAG10427.1; Synechocystis sp. PCC 6803, BAA17848.1; Anabaena variabilis PCC 29413, YP_325286.1; Nostoc sp. PCC 7120, BAB73532.1; Gloeobacter violaceus PCC 7421, NP 924690.1; Synechococcus sp. JA-33-Ab, YP_473801.1; Brevibacterium linens, AAF65581.1; Corynebacterium glutamicum, AAK64298.1. The tree is drawn to scale, with branch lengths in the same units as those of the evolutionary distances used to infer the phylogenetic tree. The evolutionary distances correspond to the number of amino acid substitutions per site and were computed using the Poisson correction method. Numbers at nodes indicate bootstrap values calculated over 500 replicates

were transformed with plasmid pSI105-psy and the transformants were firstly selected on the basis of their paromomycin resistance. The colonies obtained after transformation that showed resistance to paromomycin were screened for the insertion of $C z P S Y$ cDNA in their genome by PCR test. Figure 6 shows some of the positive transformants analysed exhibiting a band of $147 \mathrm{bp}$, which correspond to the $C z P S Y$ cDNA integrated in their genome. The primers used for PCR analysis to confirm this integration were the same used for the expression analysis of $C z P S Y$ (Table 1). More than 100 colonies resistant to paromomycin were analysed and $80 \%$ of them were found positives. Some of these positive transformants selected by PCR were grown in liquid TAP medium and their carotenoids content analysed by HPLC and the relative mRNA levels of $C$. reinhardtii PSY $(C r P S Y)$ and $C z P S Y$ genes determined by qRT-PCR.

Analysis of carotenoids content and mRNA levels of $C r P S Y$ and $C z P S Y$ genes in both parental and $C z P S Y$-transformed strains of $C$. reinhardtii

The mRNA relative abundance of endogenous ( $\mathrm{CrPSY}$ ) and exogenous $(C z P S Y)$ phytoene synthase genes of parental and $C z P S Y$-transformant strains of $C$. reinhardtii was monitored by qRT-PCR, and changes in cellular carotenoids content were determined in order to correlate transcript levels with the biosynthesis of those specific carotenoids. Figure 7 shows carotenoids content and mRNA levels of $C r P S Y$ and $C z P S Y$ genes in parental and six selected $C z P S Y$ transformants. The carotenoid profile of parental and $C z P S Y$ transformed $C$. reinhardtii cells were very similar, being violaxanthin the major carotenoid, followed by lutein and $\beta$-carotene, $\alpha$-carotene showing the lowest levels. However, some of the transformants (approximately 10\% of the positives found) exhibited a violaxanthin and lutein content of 1.8- to 2.0- and 2.0- to 2.2-fold higher than the parental strain, respectively, whereas $\beta$-carotene and $\alpha$ carotene levels were virtually identical to those of the wild type strain. T10 and T11 are two representative examples of these violaxanthin and lutein hyperproducing mutants (Fig. 7a). Other transformants showed only a slight increase

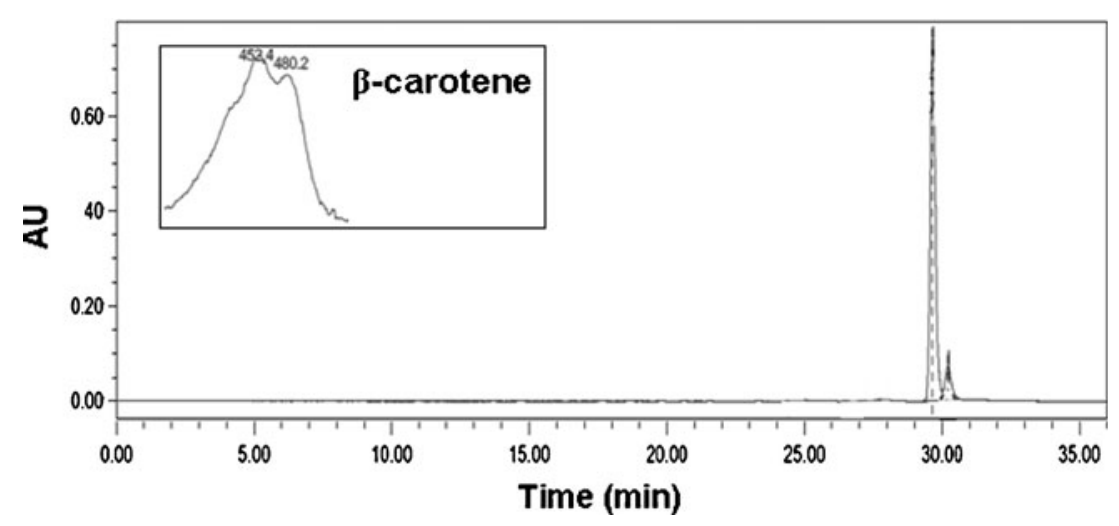

Fig. 5 HPLC elution profile of carotenoids extracted from cultures of $E$. coli carrying plasmids $\mathrm{pAC}-85 \mathrm{~b}+\mathrm{pQE}-C z P S Y$. The absorption spectrum of $\beta$-carotene is also shown. E. coli BL21 (DE3) colonies transformed with the indicated plasmids were isolated in the presence of chloramphenicol+ampicillin. Peak identification, $\beta$-carotene 

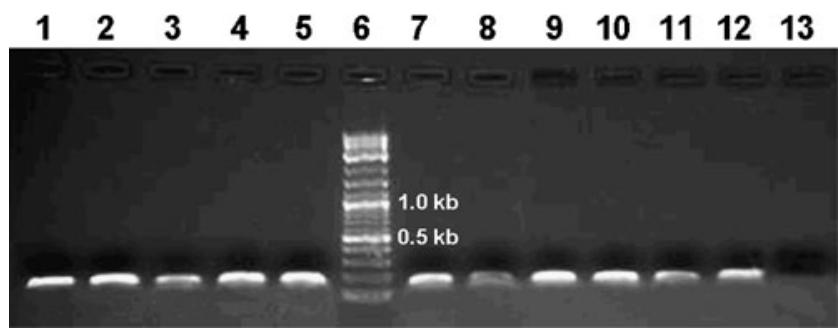

Fig. 6 Verification of the insertion of the plasmid pSI105-CzPSY in the genome of $C$. reinhardtii by PCR. C. reinhardtii cells transformed with plasmid pSI105-CzPSY were grown in TAP medium with paromomycin $\left(30 \mu \mathrm{g} \mathrm{mL} \mathrm{m}^{-1}\right)$, and paromomycin-resistant colonies were tested by PCR. Line 6 is the $2-\log$ DNA ladder $(0.1-10 \mathrm{~kb}$, Biolabs). Lines 1-5 and 7-11 are transformants analysed. Lines 12 and 13 are wild strain from $C$. zofingiensis and $C$. reinhardtii, respectively

in the levels of violaxanthin and lutein, although in all of them the exogenous $P S Y$ gene was adequately transcribed and high levels of $C z P S Y$ mRNA were found.

Regarding the expression analysis, in all the transformants analysed the levels of $C z P S Y$ mRNA were higher than those of the endogenous PSY, since exogenous $C Z P S Y$ was constitutively expressed under the control of the strong promoters $r b c S 2$ and $h s p 70 A$. Transformants $\mathrm{T} 1$ and $\mathrm{T} 10$ showed the highest $C z P S Y$ levels, which reached 140-fold the level of the endogenous PSY. Expression levels of endogenous $P S Y$ in transformants were quite similar to that of the wild type cells. Levels of PSY transcripts were standardized respect to the housekeeping control gene $(C B L P)$ and expressed as numbers of normalized molecules.

\section{Discussion}

Phytoene synthase catalyzes the first step of the carotenoid biosynthetic pathway and is considered as a rate-limiting key enzyme in this pathway (Cunningham and Gantt 1998) and as the branching enzyme that determines the carbon flux towards carotenoids production (Shewmaker et al. 1999). Therefore, PSY has been the target of genetic manipulation in many crop plants to increase carotenoid biosynthesis (Fray et al. 1995; Fraser et al. 2002; Lindgren et al. 2003). The $C z P S Y$ gene isolated in this work, as well as the known $P S Y$ genes of eukaryotic microalgae, have a smaller number of exons than the common five or seven exons in plants. Southern blot analysis has demonstrated that in $C$. zofingiensis, as in other microalgae as $C$. reinhardtii and D. salina (McCarthy et al. 2004; Lohr et al. 2005; Yan et al. 2005), only a single gene coding for PSY is present. However, recently, it has been described that other algae as Dunaliella bardawil and Micromonas pusilla contain either multiple paralogous or orthologous copies of the $P S Y$, which could be expected that analogous to the diversity of $P S Y$ genes and their differential a

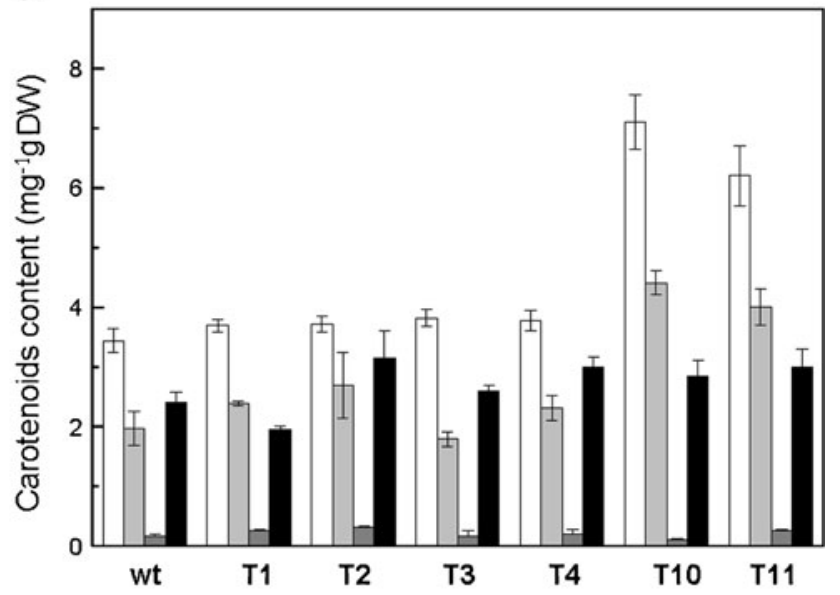

b

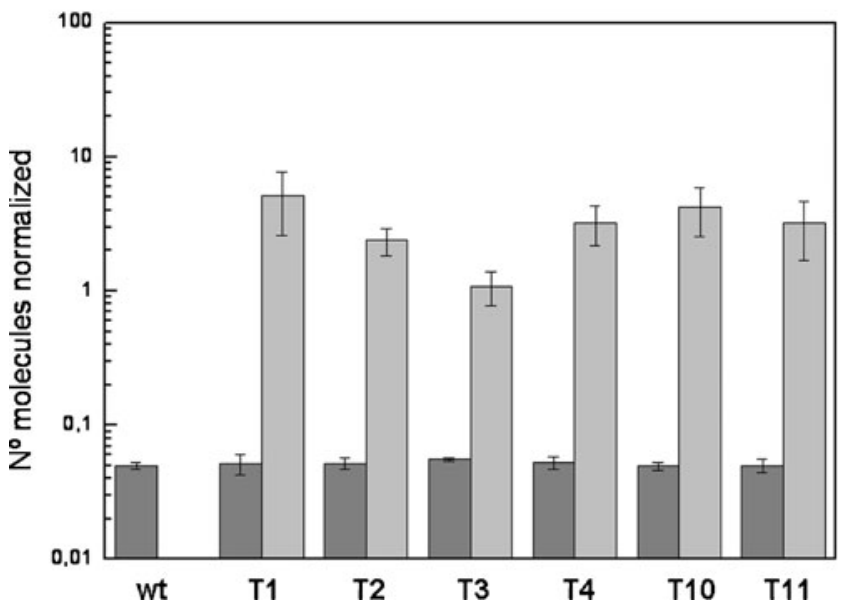

Fig. 7 Carotenoids content (a) and mRNA relative abundance of endogenous $C r P S Y$ and foreign $C z P S Y$ (b) in cells of $C$. reinhardtii wild type (wt) and six selected transformants $(T 1, T 2, T 3, T 4, T 10$, and T11). a Violaxanthin (white bar), lutein (light-grey bar), $\alpha$-carotene (darkgrey bar), and $\beta$-carotene (black bar). b Levels of PSY transcripts were normalized respect to the housekeeping control gene $(C B L P)$. Endogenous CrPSY (dark-grey bar); foreign CzPSY (light-grey bar). Error bars indicate the standard deviations of four independent measurements

expression in higher plants, some algae also differentially could regulate expression of their multiple copies of PSY gene. The comparative analyses of various algal genomes for PSY in combination with phylogenetic analyses have suggested an ancient gene duplication creating two classes of PSY (Tran et al. 2009). Our results indicate that only one class of PSY seems to be present in C. zofingiensis. Alignments at the protein level have indicated that CzPSY has the essential characteristics of both classes of PSY including predicted substrate- $\mathrm{Mg}^{2+}$-binding sites (Aspartaterich regions) and catalytic residues (data not shown). Moreover, the alignments have showed that the sequence differences between plant and bacterial PSY are mainly found at the $\mathrm{N}$ terminus due to the presence of a signal 
peptide responsible for the localization of these enzymes in chloroplasts and chromoplasts (Cunningham and Gantt 1998). The high homology degree found between the predicted protein encoded by the new gene isolated from C. zofingiensis and plant phytoene synthases, specially with the known PSY of other green microalgae, would have been probably enough to consider this new gene as a PSY, but functional analysis has definitely confirmed this hypothesis. It has been shown by functional complementation in $E$. coli that this gene encodes phytoene synthase. Although the environment and the processing of the putative PSY gene in the prokaryotic system probably differ from those in the algal original system, the obtained gene product was functional and able to catalyze the synthesis of phytoene, complementing plasmid pAC85 and resulting in the accumulation of $\beta$ carotene (Fig. 5). This confirms that the isolated gene is responsible for the synthesis of phytoene from two GGPP molecules.

Overexpression of bacterial or plant PSY genes in crop plants has resulted in increases in total carotenoid content of about 1.8-6.3-fold in carrot roots (Hauptmann et al. 1997), tomato fruit (Fraser et al. 2002), and potato tubers (Ducreux et al. 2005). Higher carotenoids increases have been reported in plant or tissues with no carotenoids or with very original levels (Farré et al. 2010), such a 50-fold increase in canola seeds (Shewmaker et al. 1999). Microalgae combine the fast and easy growth of bacteria with an active isoprenoid metabolism that ensures enough precursors for carotenogenic pathway and adequate storage capacity. The unicellular microalgae $C$. reinhardtii is the first and best studied transformed chlorophyta. It grows at high rates under photoautotrophic, heterotrophic or mixotrophic conditions and its nuclear genetic manipulation is easy and well established. All these reasons make $C$. reinhardtii a good candidate to express foreign carotenogenic genes, for both carrying out basic metabolic and regulatory studies of the pathway as well as for the biotechnological production of commercially interesting carotenoids. As the sequence of the $P S Y$ from $C$. zofingiensis shares a $76 \%$ of identity with that reported for Chlamydomonas PSY, the expression of such a gene in the nuclear genome of this microalga is a step forward both in the characterization of the gene and in the increased production of interesting carotenoids in microalgae. In this study, the nuclear transformation of $C$. reinhardtii with $C z P S Y$ gene resulted in a significant accumulation of violaxanthin and lutein content that reached 2.0- and 2.2fold, respectively, as compared with the parental level (Fig. 7a). Moreover, transformants showed an overexpression of $C$. zofingiensis PSY gene due to the strong promoters used (Fig. $7 \mathrm{~b}$ ). These results suggest that the higher carbon flux from GGPP to carotenoid synthesis was promoted by a combination of the expression of $C z P S Y$ gene product in $C$. reinhardtii as well as the expression of the endogenous copy of the gene. The $C z P S Y$ transcript level reached 140-fold the level of the endogenous PSY of the wild type in the transformants $\mathrm{T} 1$ and $\mathrm{T} 10$, however the violaxanthin and lutein contents in these transformants were only 2.0- and 2.2-fold higher than the parental strain, respectively, in $\mathrm{T} 10$ transformant, and $7 \%$ and $21 \%$ higher, respectively, in T1. Although transcriptional control has been shown to be the most important regulation factor for carotenogenic genes, a possible explanation for these results is that post-transcriptional and translational controls play also important roles, as wells as the stability of RNA, since silencing of the $P S Y$ endogenous gene by artificial miRNAs has been described in Chlamydomonas (Molnar et al. 2009). Moreover, an increase in the levels of PSY cannot be directly correlated with an increase in carotenoids content, since the protein could not be correctly targeted, processed or assembled into a fully functional complex. In transgenic tomato and potato plants expressing a bacterial phytoene synthase (CRTB), despite CRTB enzyme activity being substantially elevated, there was only a moderate increase in total carotenoid content and no linear correlation between the levels of transcript, protein, enzyme activity, and total carotenoids (Fraser et al. 2002; Ducreux et al. 2005).

Overexpression of an exogenous $P S Y$ in tomato resulted in an accumulation of phytoene, and in a decrease in the flux control coefficient for PSY. Then, the increase in PSY activity shifts probably the regulatory step from PSY to a later enzyme (Shewmaker et al. 1999; Fraser et al. 2002). Accumulation of $\beta$-carotene in higher plants overexpressing exogenous $P S Y$, probably indicates that $\beta$-carotene hydroxylases become rate-limiting as the carotenogenic flux increases (Ducreux et al. 2005). In our study no increase in the content of phytoene or other carotenoids intermediates were detected, showing that other enzymes in the pathway are not limited in Chlamydomonas, and that phytoene, $\beta$-carotene or other intermediates are not inaccessible to the downstream enzymes of the pathway in the single cell of Chlamydomonas. Phytoene was consecutively converted to the downstream metabolites $\alpha$-carotene, lutein, $\beta$-carotene and violaxanthin, catalyzed by endogenous carotenoid biosynthetic enzymes such as PDS, ZDS, LCYb, LCYe, CHYb, and ZEP.

In plants and microalgae, carotenoid biosynthesis is part of the plastidic terpenoid metabolim. GGPP is a common intermediate to the different terpenoid biosynthetic pathway such as carotenoids, chlorophylls, gibberellins and quinones. The engineering of any terpenoid pathway may have a direct effect on other branches of the pathway. In the case of carotenoid overproduction, the increase of carbon flux into the carotenoid pathway can produce limitations especially in the synthesis of gibberellins, chlorophylls, 
and quinones with negative effects on growth and photosynthesis, respectively. In some higher plants overexpressing PSY genes undesired collateral effects or low carotenoids increase were observed (Farré et al. 2010). The constitutive expression of phytoene synthase genes in tomato (Lycopersicum esculentum) and tobacco (Nicotiana tabacum) resulted in dwarfism and chlorosis in the plants (Fray et al. 1995; Busch et al. 2002) correlated with a decrease of gibberellins. But so far, in our case, no atypical phenotype was observed in the Chlamydomonas transformants, showing even a similar growth rate than the wild type cultures (data not shown).

In conclusion, $C$. zofingiensis is a model green microalga to study the regulation of the carotenoid biosynthetic pathway, since it accumulates both lutein and astaxanthin. In this study the phytoene synthase gene from $C$. zofingiensis has been isolated and functionally characterized. The overexpression of CzPSY in Chlamydomonas cells under the control of strong constitutive promoters has resulted in a significant enhancement in the content in violaxanthin and lutein, reaching 2.0- and 2.2-fold the values of control untransformed cells, respectively. This is a successful case of manipulation of the carotenogenic pathway in eukaryotic microalgae, which opens up the possibility of enhancing the productivity of microalgalbased systems to produce carotenoids and offers an excellent tool to gain basic knowledge about an important pathway that at present is not yet completely characterized and a promising alga for industrial applications.

Acknowledgments This study was supported by a financial grant from Ministerio Español de Educación y Ciencia (AGL 2007-65303CO2), and Junta de Andalucía, group BIO-299. The authors are grateful to Prof. F. Cunningham for providing pAC-85b plasmid. We also would like to thank Marco Mejías and Carlos Parejo for their excellent technical assistance. Finally, we would like to thank to Dr. Irina Obraztsova for her help in the design of some primers.

Open Access This article is distributed under the terms of the Creative Commons Attribution Noncommercial License which permits any noncommercial use, distribution, and reproduction in any medium, provided the original author(s) and source are credited.

\section{References}

Anwaruzzaman N, Chin BL, Li XP, Lohr M, Martínez DA, Niyogi KK (2004) Genomic analysis of mutants affecting xanthophyll biosynthesis and regulation of photosynthetic light harvesting in Chlamydomonas reinhardtii. Photosynth Res 82:265-276

Arnon DI, McSwain BD, Tsujimoto HY, Wada K (1974) Photochemical activity and components of membrane preparations from blue-green algae.I. Coexistence of two photosystems in relation to chlorophyll a and removal of phycocyanin. Biochim Biophys Acta 357:231-245

Baranski R (2008) Genetic transformation of carrot (Daucus carota) and other apiaceae species. Trans Plant J 2(1):18-38
Busch M, Seuter A, Hain R (2002) Functional analysis of the early steps of carotenoid biosynthesis in tobacco. Plant Physiol 128:439-453

Caboche M (2003) A prediction service for identifying putative Nterminal targeting sequences. Available at: http://urgi.versailles. inra.fr/predotar/predotar.html. Accessed 15 October 2003

Chen Y, Li F, Wurtzel ET (2010) Isolation and characterization of the $Z$-ISO gene encoding a missing component of carotenoid biosynthesis in plants. Plant Physiol 153:66-79

Cordero BF, Obraztsova I, Martín L, Couso I, León R, Vargas MA, Rodríguez H (2010) Isolation and characterization of a lycopene $\beta$-cyclase gene from the astaxanthin-producing green alga Chlorella zofingiensis (Chlorophyta). J Phycol 46:1229-1238

Cunningham FX Jr, Gantt E (1998) Genes and enzymes of carotenoid biosynthesis in plants. Annu Rev Plant Physiol Plant Mol Biol 49:55-583

Cunningham FX Jr, Gantt E (2005) A study in scarlet: enzymes of ketocarotenoid byosinthesis in the flowers of Adonis eastivalis. Plant Cell 41:478-492

Cunningham FX Jr, Gantt E (2007) A portfolio of plasmids for identification and analysis of carotenoid pathway enzymes: Adonis aestivalis as a case study. Photosynth Res 92:245-259

Cunningham FX Jr, Pogson B, Sun Z, McDonald KA, DellaPenna D, Gantt E (1996) Functional analysis of the $\beta$ - and $\varepsilon$-lycopene cyclase enzymes of Arabidopsis reveals a mechanism for control of cyclic carotenoid formation. Plant Cell 8:1613-1626

Del Campo JA, Rodríguez H, Moreno J, Vargas MA, Rivas J, Guerrero MG (2004) Accumulation of astaxanthin and lutein in Chlorella zofingiensis (Chlorophyta). Appl Microbiol Biotechnol 64:848-854

Demming-Adams B, Adams WW III (2002) Antioxidants in photosynthesis nutrition. Science 298:2149-2153

Ducreux JM, Morris WL, Hedley PE, Shepherd T, Davies HV, Millan S, Taylor MA (2005) Metabolic engineering of high carotenoids potato tubers containing enhanced levels of $\beta$-carotene and lutein. J Exp Bot 56:81-89

Dweyer JH, Navab M, Dwyer KM, Hassan K, Sun P, Shircore A, Hama-Levy S, Hough G, Wang X, Drake T, Merz NB, Fogelman AM (2001) Oxygenated carotenoid lutein and the progression of early atherosclerosis. Circulation 103:2922-2927

Emanuelsson O, Nielsen H, von Hejne G (1999) ChloroP, a neural network-based method for predicting chloroplast transit peptides and their cleavage sites. Protein Sci 8:978-984

Fan L, Vonshak A, Rachel G, Hirshberg J, Cohen Z, Boussiba S (1995) The biosynthetic pathway of astaxanthin in a green alga Haematococcus pluvialis as indicated by inhibition with diphenylamine. Plant Cell Physiol 36:1519-1524

Farré G, Sanahuja G, Naqvi S, Bai C, Capell T, Zhu C, Christou P (2010) Travel advice on the road to Carotenoids in plants. Plant Sci 179:28-48

Frank HA, Cogdell RJ (1996) Carotenoids in photosynthesis. Photochem Photobiol 63(3):257-264

Fraser PD, Römer S, Shipton CA, Mills PB, Kiano JW, Misawa N, Drake RG, Schuch W, Bramley PM (2002) Evaluation of transgenic tomato plants expressing an additional phytoene synthase in a fruit specific-manner. Proc Natl Acad Sci USA 99:1092-1097

Fray W, Fraser PD, Valero D, Hedden P, Bramley PM, Grierson D (1995) Constitutive expression of a fruit phytoene synthase gene in transgenic tomatoes causes dwarfism by redirecting metabolites from the gibberellin pathway. Plant J 8:693-701

Gasteiger E, Hoogland C, Gattiker A, Duvaud S, Wilkins MR, Appel RD, Bairoch A (2005) Protein identification and analysis tools on the ExPASy server. In: Walker J, Walker M (eds) The proteomics protocols handbook. Humana Press, Totowa, pp 571-607

Gorman DS, Levine RP (1965) Cytocrome f and plastocyanin: their sequence in the photosynthetic electron transport chain of 
Chlamydomonas reinhardtii. Proc Natl Acad Sci USA 54:16651669

Gruber H, Goetinck SD, Kirk DL, Schmitt R (1992) The nitrate reductase-encoding gene of Volvox carteri: map location, sequence and induction kinetics. Gene 120:75-83

Guerin M, Huntley ME, Olaizola M (2003) Haematococcus astaxanthin applications for human health and nutrition. Trends Biotechnol 21:210-216

Hauptmann R, Eschenfeldt WH, English J, Brinkhaus FL (1997) Enhanced carotenoid accumulation in storage organs of genetically engineered plants. US Patent 5:618-988

Huang JC, Wang Y, Sandmann G, Chen F (2006) Isolation and characterization of a carotenoid oxygenase gene from Chlorella zofingiensis (Chlorophyta). Appl Microbiol Biotechnol 71:473-479

Huang JC, Liu J, Li Y, Chen F (2008) Isolation and characterization of the phytoene desaturase gene as a potential selective marker for genetic engineering of the astaxanthin-producing green alga Chlorella zofingiensis (Chlorophyta). J Phycol 44:684-690

Kim J, Smith JJ, Tian L, DellaPenna D (2009) The evolution and function of carotenoid hydroxylases in Arabidopsis. Plant Cell Physiol 50:463-479

Kindle KL (1990) High-frequency nuclear transformation of Chlamydomonas reinhardtii. Proc Natl Acad Sci USA 87:1228-1232

León R, González D, Galván A, Fernández E (2004) Transgenic microalgae as green cell factories. Trends Biotechnol 22(1):45-52

León R, Vila M, Herranz D, Vilches C (2005) Production of phytoene by herbicide-treated microalga Dunaliella bardawil in two phase systems. Biotechnol Bioeng 92:695-701

León R, Couso I, Fernández E (2007) Metabolic engineering o ketocarotenoids biosynthesis in the unicellular microalga Chlamydomonas reinhardtii. J Biotechnol 130:143-152

Li Y, Sommerfeld M, Chen F (2008a) Consumption of oxygen by astaxanthin biosynthesis: a protective mechanism against oxidative stress in Haematococcus pluvialis (Chlorophyceae). J Plant Physiol 165:1783-1797

Li Y, Huang J, Sandmann G, Chen F (2008b) Glucose sensing and the mitochondrial alternative pathway are involved in the regulation of astaxanthin biosynthesis in the dark-grown Chlorella zofingiensis (Chlorophyceae). Planta 228:735-743

Lindgren LO, Stalberg KG, Höglund AS (2003) Seed-specific overexpression if an endogenous Arabidopsis phytoene synthase gene results in delayed germination and increased levels of carotenoids, chlorophyll, and abscisic acid. Plant Physiol 132:779-785

Lohr M, Im CS, Grossma AR (2005) Genome-based examination of chlorophyll and carotenoid biosynthesis in Chlamydomonas reinhardtii. Plant Physiol 138(1):490-515

Loppes R, Radoux M, Ohresser MC, Matagne RF (1999) Transcriptional regulation of the Nial gene encoding nitrate reductase in Chlamydomonas reinhardtii: effects of the various environmental factors on the expression of a reporter gene under the control of the Nial promoter. Plant Mol Biol 41:701-711

McCarthy SS, Kobayashi MV, Niyogi KK (2004) White mutants of Chlamydomonas reinhardtii are defective in phytoene synthase. Genetics 168(3):1249-1257

Molnar A, Bassett A, Thuenemann E, Schwach F, Karkare S, Ossowski S, Weigel D, Baulcombe D (2009) Highly specific gene silencing by artificial microRNAs in the unicellular alga Chlamydomonas reinhardtii. Plant J 58:165-174

Olmedilla B, Granado F, Blanco I, Vaquero M (2003) Lutein, but not $\alpha$-tocopherol, supplementation improves visual function in patients with age-related cataracts: a 2-y double blind, placebocontrolled study. Nutrition 19:21-25
Paine JA, Shipton CA, Chaggar S, Howell RM, Kennedy MJ, Vernon G, Susan Y, Wright SY, Hinchliffe E, Adams JL, Aron L, Silverstone AL, Drake R (2005) Improving the nutritional value of Golden Rice through increased pro-vitamin A content. Nat Biotechnol 23:482-487

Sambrook J, Fritsch EF, Maniatis T (1989) Molecular cloning. A laboratory manual. Cold Spring Harbor Laboratory Press, New York

Sandmann G, Römer S, Fraser PD (2006) Understanding carotenoid metabolism as a necessity for genetic engineering of crop plants. Metab Eng 8:291-302

Shewmaker CK, Sheehy JA, Daley M, Colburn S, Ke DE (1999) Seed-specific overexpression of phytoene synthase: increase in carotenoids and other metabolic effects. Plant J 20:401-412

Sizova I, Fuhrman M, Hegemann P (2001) A Streptomyces rimosus aphVIII gene coding for a new type phosphotransferase provides stable antibiotic resistance to Chlamydomonas reinhardtii. Gene 277:221-229

Stahl W, Sies H (2005) Bioactivity and protective effects of natural carotenoids. Biochim Biophys Acta 1740:101-107

Steinbrenner J, Sandmann G (2006) Transformation of the green alga Haematococcus pluvialis with a phytoene desaturase for accelerated astaxanthin biosynthesis. Appl Environ Microbiol 72:7477-7484

Sun G, Zhang X, Sui Z, Mao Y (2007) Inhibition of pds gene expression via the RNA interference approach in Dunaliella salina (Chlorophyta). Mar Biotechnol 10(3):219-226

Sun N, Wang Y, Huang JC, Chen F (2008) Sugar-based growth, astaxanthin accumulation and carotenogenic transcription of heterotrophic Chlorella zofingiensis (Chlorophyta). Process Biochem 43:1288-1292

Tamura K, Dudley J, Nei M, Kumar S (2007) MEGA4: Molecular Evolutionary Genetics Analysis (MEGA) software version 4.0. Mol Biol Evol 24:1596-1599

Tran D, Haven J, Qiu WG, Polle JEW (2009) An update on carotenoid biosynthesis in algae: phylogenetic evidence for the existence of two classes of phytoene synthase. Planta 229:723-729

Varkonyi Z, Masamoto K, Debreczeny M, Zsiros O, Ughy B, Gombos Z, Domonkos I, Farkas T, Wada H, Szalontai B (2002) Low-temperature-induced accumulation of xanthophylls and its structural consequences in the photosynthetic apparatus of the cyanobacterium Cylindrospermopsis raciborskii: an FTIR spectroscopic study. Proc Natl Acad Sci USA 99:2410-2415

Vila M, Couso I, León R (2007) Carotenoid content in mutants of the chlorophyte Chlamydomonas reinhardtii with low expression levels of phytoene desaturase. Process Biochem 43(10):11471152

von Heijne G (1992) Membrane protein structure prediction: hydrophobicity analysis and the 'Positive inside' rule. J Mol Biol 225:487-494

von Kampen J, Nieländer U, von Wettern M (1994) Stress-dependent transcription of a gene encoding a Gb-like polypeptide from Chlamydomonas reinhardtii. J Plant Physiol 143:756-758

Yan Y, Zhu YH, Jiang JG, Song DL (2005) Cloning and sequence analysis of the phytoene synthase gene from a unicellular Chlorophyte, Dunaliella salina. J Agric Food Chem 53:14661469

Zhang J, Tao N, Xu Q, Zhou W, Cao H, Xu J, Deng X (2009) Functional characterization of citrus PSY gene in Hongkong kumquat (Fortunella hindsii Swingle). Plant Cell Rep 28:17371746 